\title{
$\beta$ cell replication is the primary mechanism for maintaining postnatal $\beta$ cell mass
}

\author{
Senta Georgia and Anil Bhushan
} Department of Biochemistry and Molecular Biology, University of Southern California, Developmental Biology Program, Saban Research Institute,
Children's Hospital Los Angeles, Los Angeles, California, USA.

\begin{abstract}
The endocrine pancreas undergoes major remodeling during neonatal development when replication of differentiated $\beta$ cells is the major mechanism by which $\beta$ cell mass is regulated. The molecular mechanisms that govern the replication of terminally differentiated $\beta$ cells are unclear. We show that during neonatal development, cyclin D2 expression in the endocrine pancreas coincides with the replication of endocrine cells and a massive increase in islet mass. Using cyclin $\mathrm{D2}^{-/-}$mice, we demonstrate that cyclin $\mathrm{D} 2$ is required for the replication of endocrine cells but is expendable for exocrine and ductal cell replication. As a result, 14-day-old cyclin $\mathrm{D2}^{-/-}$mice display dramatically smaller islets and a 4-fold reduction in $\beta$ cell mass in comparison to their WT littermates. Consistent with these morphological findings, the cyclin $\mathrm{D2}^{-/-}$mice are glucose intolerant. These results suggest that cyclin D2 plays a key role in regulating the transition of $\beta$ cells from quiescence to replication and may provide a target for the development of therapeutic strategies to induce expansion and/or regeneration of $\beta$ cells.
\end{abstract}

\section{Introduction}

The $\beta$ cell mass plays an essential role in determining the amount of insulin that is secreted to maintain the body's glucose levels within a narrow range. Increasing evidence suggests that the $\beta$ cell mass is dynamic and variations in insulin demand can lead to rapid and marked changes in the $\beta$ cell mass (1-3). The mass of $\beta$ cells is governed by balancing $\beta$ cell growth (differentiation and replication) and $\beta$ cell death (apoptosis). The molecular basis of the parameters regulating $\beta$ cell mass are not clear (4). Understanding the regulation of $\beta$ cell mass is especially important because the inability of the endocrine pancreas to compensate for the changing insulin demand can contribute to the pathogenesis of diabetes (5-7).

During mouse embryogenesis, $\beta$ cells are generated from a population of pancreatic progenitor cells $(8,9)$. The $\beta$ cells that differentiate from progenitor cells are postmitotic, and direct lineage tracing analyses indicate that a population of progenitor cells persists throughout embryogenesis to allow the differentiation of new $\beta$ cells $(10,11)$. During postnatal development, however, replication of differentiated $\beta$ cells can lead to addition of new $\beta$ cells (12). High rates of $\beta$ cell replication during the neonatal period results in a massive increase in $\beta$ cell mass $(13,14)$. How do terminally differentiated $\beta$ cells transition between quiescence to replication in the neonatal period? Here we examine the role of $\mathrm{D}$-cyclins in regulating cell cycle progression of $\beta$ cells in postnatal development. The three D-cyclins (cyclins D1, D2, and D3) are key components of the cell cycle machinery encoded by separate genes, but show significant homology at the protein level (15). The levels of D-cyclins are controlled by mitogens and, upon induction, associate with partner cyclin-dependent kinases CDK4 and CDK6 to drive cells into S-phase $(16,17)$. Here we report that one D-cyclin, cyclin $D 2$, is uniquely required for $\beta$ cell replication and the proper

Nonstandard abbreviations used: $\mathrm{DAB}, 3,3$ diamino benzidine; E, embryonic day; GTT, glucose tolerance test; P, postnatal day.

Conflict of interest: The authors have declared that no conflict of interest exists.

Citation for this article: J. Clin. Invest. 114:963-968 (2004)

doi:10.1172/JCI200422098. expansion of $\beta$ cell mass during postnatal development. We propose that cyclin D2 plays a crucial role within $\beta$ cells in mediating mitogenic stimuli to regulate $\beta$ cell mass in the pancreas.

\section{Results}

To establish which D-cyclins are expressed in the developing pancreas, we carried out RT-PCR on RNA isolated from the developing embryonic pancreas and neonatal pancreas. RT-PCR analysis showed high levels of cyclin D2 expression and lower levels of cyclin $D 3$ in the developing pancreas (Figure 1A). Immunohistochemistry analysis of D-cyclins in the embryonic pancreas showed that cyclin D2 expression was confined to the nucleus of pancreatic epithelial cells and was absent in differentiated endocrine cells (Figure 1B). Consistent with the RT-PCR analysis, low levels of cyclin D3 expression was observed in the pancreas during embryonic development (data not shown). Analysis of pancreata from the postnatal period confirmed the complete absence of cyclin D1 expression (Figure 1C) but showed that the remaining D-cyclins were expressed in a dynamic fashion, often in mutually exclusive cell types. Cyclin D3 expression was restricted to exocrine and ductal cells and was not present in the endocrine cells (Figure 1D). Cyclin D2, on the other hand, was expressed in the endocrine pancreas as well as the exocrine and ductal tissue (Figure 1, E and F). Costaining of cyclin D2 with insulin demonstrated that cyclin D2 was expressed in the nuclei of a subset of $\beta$ cells within islets (Figure 1, E and F). Cyclin D2 expression was most often seen in larger islets and rarely observed in small clusters of endocrine cells or ductal cells that stain positive for endocrine hormones (Figure $1 \mathrm{~F})$. The proportion of the $\beta$ cells expressing cyclin D2 increased during the first week after birth and subsequently decreased. As a result, relatively few cyclin D2-positive $\beta$ cells were visible at the end of the second week (Figure 1G). Cyclin D2 also decreased in exocrine and ductal tissue, and by postnatal day 14 (P14) these tissues no longer expressed cyclin D2 (Figure 1H), but continued to express cyclin D3 (data not shown).

To address whether cyclin D2 was required for endocrine pancreatic development, we analyzed mice in which the cyclin D2 


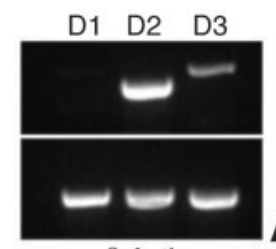

$\beta$ Actin
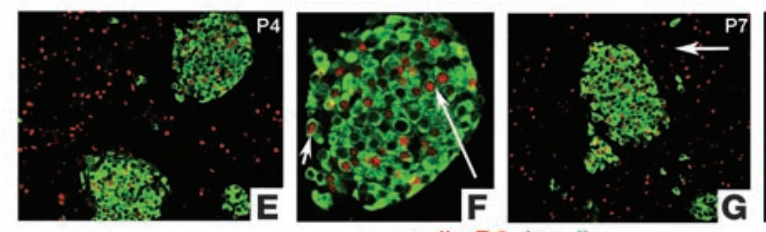

cyclin D2 insulin
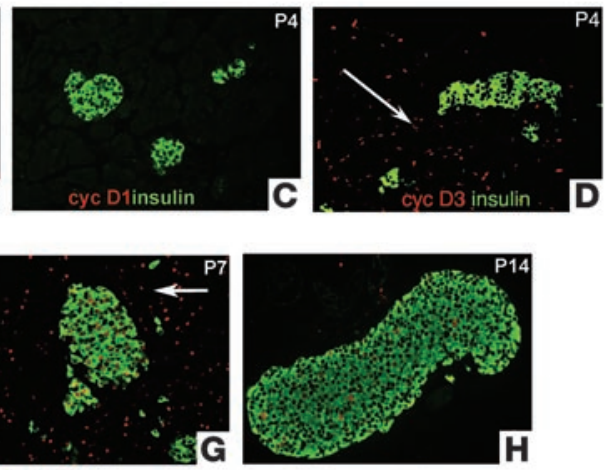

Figure 1

Cyclin D2 expression during pancreatic development. We carried out RT-PCR on RNA isolated from embryonic pancreata and stained pancreatic sections from various ages (as indicated) with anti-glucagon (green), anti-insulin (green), and anti-D-cyclin (red; as indicated) Ab's. (A) Expression of cyclins D1, D2, and D3 in the developing pancreas by RT-PCR. RNA was isolated from E12.5 pancreata. (B) Cyclin D2 (cyc D2) expression in progenitor cells during early embryonic development. At E11.5, epithelial progenitor cells but not differentiated cells express cyclin D2. The dorsal pancreatic bud is outlined. At this stage, cyclin D2 expression is restricted to the epithelium and is not observed in the mesenchyme. (C) At P4, cyclin D1 (cyc D1) expression is not observed in the pancreas. (D) Cyclin D3 (cyc D3) is expressed in the exocrine and ductal (arrow) tissue but excluded from the endocrine pancreas. (E) Cyclin D2 is expressed throughout the pancreas at P4. Costaining with insulin Ab shows that cyclin D2 is expressed in the islets. At this stage, $34.5 \% \pm 2.0 \%$ of islet cells express cyclin D2. (F) Higher magnification of an islet from $\mathbf{E}$ showing cyclin D2 expression is localized to the nucleus of $\beta$ cells (arrows). (G) Cyclin D2 continues to be expressed in the endocrine pancreas at $\mathrm{P} 7(33.2 \% \pm 4 \%$ of islet cells) and in the ductal tissue (arrow). (H) By P14, few cells in the endocrine pancreas express cyclin D2 (11.3\% $\pm 1.4 \%$ of islet cells). Exocrine and ductal tissues do not show any expression of cyclin D2 at this stage.

gene had been inactivated by homologous recombination in ES cells. Because cyclin D2 is expressed in pancreatic progenitor cells during embryonic development, we investigated whether cyclin D2 played a role in coordinating the proliferation of progenitor cells with differentiation of endocrine cells. Histological and immunofluorescence analysis revealed no significant differences in islet structure, composition, or endocrine mass in the pancreas from late gestation (embryonic day [E]17.5) cyclin $D 2^{-/-}$embryos compared with WT littermates (data not shown). Thus, cyclin D2 appeared to be dispensable for the formation of the endocrine pancreas during embryonic development.

To determine whether cyclin D2 could play a role in the development of the endocrine pancreas in the postnatal period, we examined the morphology of islets in pancreata from mice 8 weeks after birth. $\mathrm{H} \& \mathrm{E}$ staining revealed a striking reduction in the size of islets in the pancreata from cyclin $\mathrm{D}^{-/-}$mice as compared with their littermates (Figure 2, A and B). Immunohistological analysis of insulin and glucagon expression in the pancreatic tissue showed that the smaller islets expressed these hormones (Figure 2, C and D). No abnormal extraislet staining using a cocktail of islet hormone Ab's was visible in the pancreata from cyclin D2/- mice (data not shown). In 6-week-old mice, the mean islet diameter of WT mice was $202 \pm 13$ $\mu \mathrm{m}$ versus $76.5 \pm 4 \mu \mathrm{m}$ for the cyclin $\mathrm{D} 2^{-/-}$mice $(P<0.0001)$. The islets in cyclin D2-/- mice retained the characteristic distribution of endocrine cells with $\beta$ cells forming the core and $\alpha$ cells at the periphery forming the mantle. No necrosis or fibrosis was apparent in the cyclin $\mathrm{D}^{-/-}$pancreata and the size and weight of these pancreata were similar to the WT littermates. The size of individual $\beta$ cells did not $\mathbf{H}$

appear to differ in the cyclin $D 2^{-/-}$and WT littermates, indicating that the reduced islet size was likely due to a decrease in the number of $\beta$ cells. Because the number of endocrine cells depends on the balance of neogenesis, replication, and apoptosis of endocrine cells, we considered whether any of these mechanisms were affected by the absence of cyclin D2.

A number of observations, including prevalence of hormone-positive ductal cells as well as the close proximity of islets to ducts, have led to the idea that endocrine cell differentiation occurs in the ducts (12). In WT neonates, hormone-positive staining of ductal cells was rarely observed right after birth, but was more frequently observed by the end of the first week of postnatal development (Figure 2E). In cyclin D2-/- P7 mice, hormone-positive ductal cells were clearly visible at roughly similar frequencies as in WT littermates (Figure 2F). In addition, the distribution of extraislet cells with immunoreactive insulin was similar in WT and cyclin D2 $2^{-/}$mice. These results indicate that the frequency of hormonepositive ductal cells was unaffected by the absence of cyclin D2. We also did not observe any difference in the levels of apoptosis in the endocrine pancreas in WT and cyclin $D 2^{-/-}$mice (data not shown).

We examined the replicative state of $\beta$ cells during the postnatal period by assessing their ability to incorporate BrdU. BrdU, a thymidine analogue, is incorporated in the nucleus of cycling cells during the S-phase of the cycle. Throughout the first week after birth, pancreata from WT neonates showed a large number of cells within islets stained for BrdU. Costaining with insulin confirmed that a large proportion of $\beta$ cells underwent replication in the early postnatal period (Figure 3A). Strikingly, no incorporation of BrdU was observed in $\beta$ cells in the pancreata from cyclin $D 2^{-/-}$littermates (Figure $3 \mathrm{~B}$ ). Comparison of the replicative index in WT versus cyclin D2 $\%$ - P4 neonates revealed a marked defect in $\beta$ cell replication in cyclin $\mathrm{D}^{-/-}$mice $(9.2 \%$ versus $0 \%$, $P<0.001)$. To test whether cyclin D2 plays a role in the replication of other endocrine cells, we examined in these pancreata whether glucagon-stained cells also incorporated BrdU. In pancreata from WT neonates, a few glucagon-positive cells showed incorporation of $\mathrm{BrdU}$, indicating that $\alpha$ cells, in addition to $\beta$ cells, replicate and expand during early postnatal development (Figure 3C). Again, no incorporation of BrdU was observed in $\alpha$ cells in the pancreata from cyclin D2- $2^{-/}$littermates (Figure 3D). The replication defect in cyclin $\mathrm{D} 2^{-/-}$mice was restricted to the endocrine pancreas as exocrine and ductal cells that incorporated BrdU were readily visible. To further characterize replication in the pancreata of cyclin D2-/- mice, we examined the incorporation of BrdU in exocrine tissue identified by amylase staining. No difference in the BrdU incorporation in exocrine tissue was observed in the cyclin $D 2^{-/-}$pancreata compared with those from WT littermates (Figure 3, E and F). 


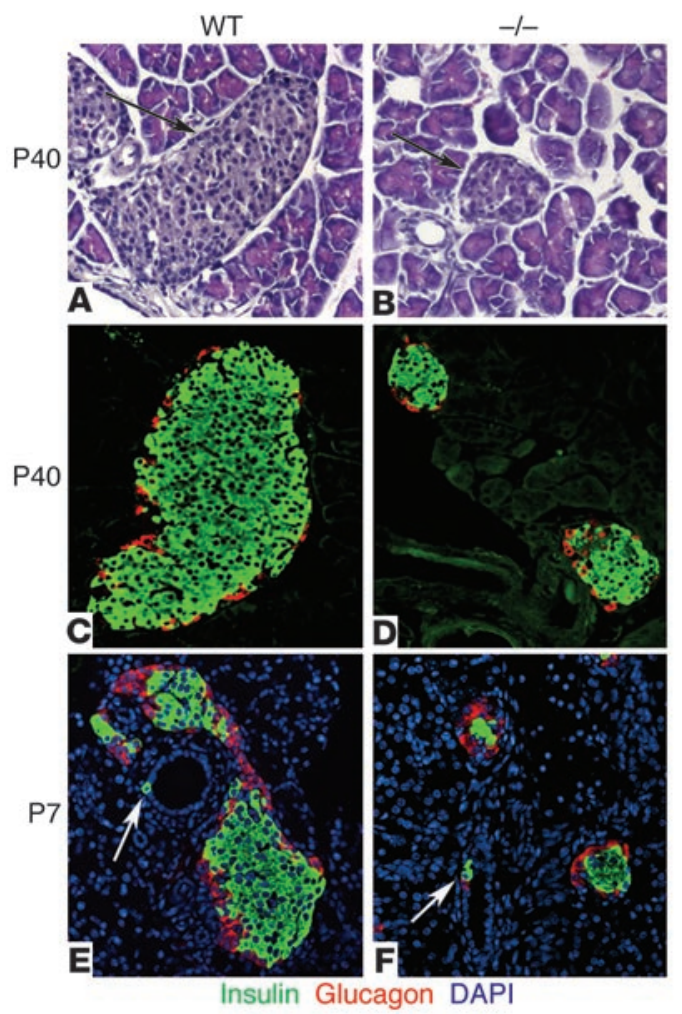

We next examined whether the highly selective defect in endocrine cell replication in the absence of cyclin D2 was due to the inability of endocrine cells to upregulate the remaining D-cyclins. We compared the expression of cyclin D1 and D3 in the pancreata of neonatal WT and cyclin D2-/- littermates. As shown earlier, in WT P4 mice, cyclin D3 was expressed in the exocrine and ductal tissue, but absent from endocrine tissue (Figure 4A). In cyclin D2-/littermates, the expression of cyclin D3 in the exocrine and ductal tissue was similar to the WT mice. Significantly, costaining with insulin demonstrated that cyclin D3 expression is not detectable in $\beta$ cells from $c y c l i n$ D2 $2^{-/-}$mice (Figure $4 \mathrm{~B}$ ). We also failed to observe

\section{Figure 2}

Islet morphology and neogenesis of endocrine cells in WT and cyclin $D 2^{-/-}$mice. We stained pancreatic sections from 6-week-old WT and cyclin D2-/- (-/-) littermates with anti-glucagon and anti-insulin Ab's. We estimated the islet size by measuring the longest axis of ten randomly selected islets that were at least ten cells wide. (A and $\mathbf{B}$ ) H\&E-stained pancreata from WT (A) and cyclin D2-/- (B) mice. The densely nucleated islets are indicated by arrows. (C) WT mice display a characteristic structure with $\alpha$ cells on the periphery and $\beta$ cells at the core of the islet. (D) The cyclin $D 2^{-/-}$mice displayed dramatically smaller islets, although the morphology of $\alpha$ cells on the periphery and $\beta$ cells at the core of the islet are preserved. The mean islet diameter of WT mice is $202 \pm 13 \mu \mathrm{m}$ versus $76.5 \pm 4 \mu \mathrm{m}$ for the cyclin $D 2^{-/-}$mice $(P<0.0001)$. (E and F) Pancreatic sections from P7 WT and cyclin $D 2^{-/-}$littermates stained with anti-insulin and anti-glucagon Ab's. DAPI nuclear stain allows for the easy identification of ductal cells based on their characteristic morphology. Insulin-positive ductal cells in WT (E) and in cyclin $\mathrm{D2}^{-{ }^{--}}$(F) littermates are shown (arrows). The frequency of hormone-positive ductal cells in the cyclin $D 2^{-1-}$ mice is similar to that of WT littermates.

any cyclin D1 expression in $\beta$ cells of the pancreas from P4 cyclin D2 $2^{-/-}$mice (Figure $\left.4 \mathrm{C}\right)$. We next examined the expression of cyclin D1 and D3 in cyclin D2 $2^{-/}$mice at the end of the postnatal remodeling phase of the endocrine pancreas. Pancreatic sections from P14 WT mice did not stain for cyclin D1 (Figure 4D). In the cyclin D2 $2^{-/-}$ littermates, however, cyclin D1 expression was clearly detectable in the endocrine pancreas. Costaining with glucagon revealed the cyclin D1-expressing cells in the pancreata from cyclin $\mathrm{D} 2^{-/-}$mice were restricted to the core of the islets (Figure 4E), and insulin staining demonstrated that cyclin D1 expression was expressed in the nuclei of $\beta$ cells (Figure 4F). Cyclin D3 expression continued to be detected in the exocrine and ductal tissue in P14 WT and cyclin D2 $2^{-/}$mice, but not in endocrine cells (data not shown).

Because $\beta$ cell replication is an important parameter that regulates $\beta$ cell mass, we quantified the total $\beta$ cell mass during the 2 -week period of postnatal development. At P4, the $\beta$ cell mass in the pancreas of cyclin $D 2^{-/-}$mice was similar to its WT littermates (WT, $0.39 \pm 0.02 \mathrm{mg}$, versus cyclin $D 2^{-/-}, 0.36 \pm 0.01 \mathrm{mg}$ ). The $\beta$ cell mass subsequently increased rapidly during postnatal develop-

\section{Figure 3}

Incorporation of BrdU during postnatal pancreatic development of WT and cyclin D2-/- mice. BrdU was injected in 4- and 7-dayold mice 2 hours before being sacrificed. (A and $\mathbf{B})$ Sections from pancreata costained with anti-insulin and anti-BrdU Ab's. (A) In P4 WT mice, a fraction of $\beta$ cells that incorporate BrdU are evident during the first week of postnatal development. Quantification of 20 representative islets showed that $9.2 \%$ of $\beta$ cells incorporated BrdU in P4 WT mice. Arrow indicates an example of an islet with BrdU-positive $\beta$ cells. (B) In cyclin D2--- littermates, $\beta$ cells that incorporated BrdU are not observed. Arrows indicate islets in cyclin D2 $2^{--}$that do not contain BrdU-positive $\beta$ cells. (C and D) Sections from pancreas costained with anti-glucagon and anti-BrdU Ab's. (C) Glucagon-positive cells incorporate BrdU in the WT pancreas. Arrow indicates an example of an islet with a BrdU-positive $\alpha$ cell. (D) No glucagon-positive cells that incorporate BrdU are observed. (E and F) Sections from pancreas costained with anti-amylase and anti-BrdU Ab's. BrdU incorporation is similar in exocrine and ductal tissue of the pancreata from WT (E) and cyclin D2-/- mice (F).

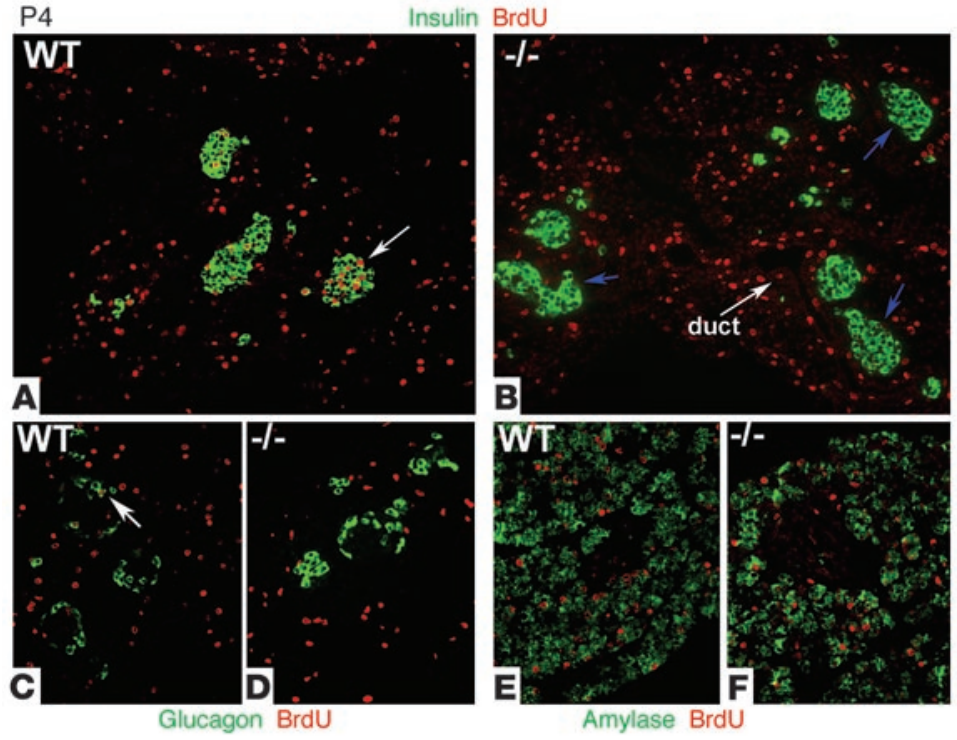




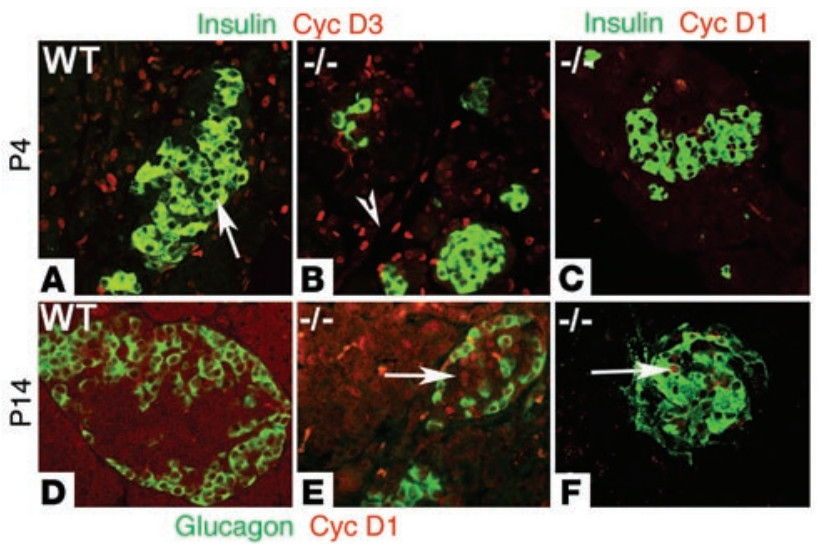

Figure 4

Expression of cyclin D1 and D3 in WT and cyclin D2-/- mice during postnatal development. (A and B) Sections from pancreata of 4-dayold WT and cyclin $D 2^{-/-}$mice were stained with anti-cyclin D3 and anti-insulin Ab's. (A) In P4 WT mice, cyclin D3 expression is observed in exocrine and ductal tissue but is not observed in the endocrine pancreas. Arrow indicates islet lacking cyclin D3 expression. (B) In cyclin $D 2^{-/-}$littermates, cyclin D3 expression is observed in exocrine and ductal tissue (arrowhead) but not observed in the $\beta$ cells. (C) Sections from pancreata of 4-day-old cyclin $D 2^{-1-}$ mice were stained with anticyclin D1 and anti-insulin Ab's. Cyclin D1 expression is not observed in the $\beta$ cells in cyclin $D 2^{-/-}$mice. (D-F) Sections from pancreata of 14-day-old WT and cyclin $\mathrm{D} 2^{-/-}$mice are stained with anti-cyclin D1, anti-glucagon (D and E), and anti-insulin (F) Ab's. (D) Cyclin D1 is not expressed in the pancreas from WT P14 mice. (E) Cyclin D1 expression is observed within islets in pancreata from cyclin $D 2^{-/-}$littermates. Arrow indicates cyclin D1 expression within an islet of P14 cyclin $D 2^{-/-}$pancreas. (F) Cyclin D1 expression is observed in the nuclei of insulin-positive cells. Arrow indicates cyclin D1 expression in a $\beta$ cell of P14 cyclin D2-/- pancreas.

ment, and a 4-fold increase in $\beta$ cell mass was apparent in P14 WT mice (Figure 5A). By contrast, no significant increase in the $\beta$ cell mass was apparent in the cyclin D2 $2^{-/}$littermates. Consequently, by $\mathrm{P} 14$, the total $\beta$ cell mass of cyclin $D 2^{-/-}$mice is about $30 \%$ of that of their WT littermates (cyclin D2- $2^{--}, 0.5 \pm 0.05 \mathrm{mg}$, versus WT, $1.5 \pm 0.16 \mathrm{mg} ; P<0.05)$. This lack of increase in mass was specific to the endocrine pancreas, because the total weight of an individual pancreas from cyclin $D 2^{-/-}$mice did not differ significantly from their WT littermates throughout postnatal development. This is consistent with the observation that exocrine tissue, which makes up $98 \%$ of the pancreas, was unaffected in the absence of

\section{Figure 5}

$\beta$ cell mass in WT and cyclin D2-/- mice during postnatal development. (A) The $\beta$ cell mass per pancreas was estimated as the product of the relative cross-sectional area of $\beta$ cells (determined by quantification of the cross-sectional area occupied by $\beta$ cells divided by the cross-sectional area of total tissue) and the weight of the pancreas. $\beta$ cell mass was measured at a postnatal age as indicated. Data are mean values \pm SEM of four mice per genotype. In WT mice, $\beta$ cell mass measurement showed a 4-fold increase by P14. In contrast, cyclin D2 $2^{-/}$littermates did not show any significant increase in $\beta$ cell mass in the same period. (B) Body weights of cyclin D2 litters were measured to correspond to the $\beta$ cell mass measurements during the postnatal period. The $c y c l i n D 2^{-/-}$mice weights do not differ from their littermates. (C) The relative $\beta$ cell mass is calculated as a ratio of the $\beta$ cell mass to body weight. Data are mean values \pm SEM of ten mice per genotype.

cyclin D2. Moreover, the cyclin $\mathrm{D} 2^{-/-}$mice did not differ from their WT littermates in body weight or size (Figure 5B). Thus, unlike WT mice, the increase in body weight of cyclin $\mathrm{D} 2^{-/-}$mice was not matched by the increment in $\beta$ cell mass (Figure 5C).

To test whether the decrease in the relative $\beta$ cell mass of cyclin D2 $2^{-/-}$mice resulted in altered serum insulin levels and abnormal glucose homeostasis, we measured serum insulin levels and performed glucose tolerance tests (GTTs) in WT and cyclin $D 2^{-/-}$mice. The serum insulin levels after overnight fasting were similar in WT and cyclin D2 $2^{-/-}$mice. After glucose challenge, however, serum insulin levels doubled in WT mice but only increased slightly in cyclin $\mathrm{D2}^{\gamma^{--}}$mice (Figure $6 \mathrm{~A}$ ). The cyclin $\mathrm{D} 2^{-{ }^{-}}$mice had fasting blood glucose levels that were consistently elevated when compared with the WT mice. The cyclin $\mathrm{D} 2^{-1-}$ mice also showed a decreased ability to clear glucose from the blood following intraperitoneal glucose injection. WT mice peaked at levels between 140 and $160 \mathrm{mg} / \mathrm{dl} 15$ minutes after injection and reached baseline by the end of the 90-minute testing period (Figure $6 \mathrm{~B})$. In contrast, cyclin $\mathrm{D} 2^{-/-}$mice had elevated blood glucose levels $(300-350 \mathrm{mg} / \mathrm{dl}$ ) within 15 minutes of injections. Moreover, the blood glucose levels failed to return to baseline levels during the testing period and remained in the range of $150-250 \mathrm{mg} / \mathrm{dl}$ 120 minutes after the injection (data not shown).

\section{Discussion}

Recent work suggests that new $\beta$ cells during adult life originate from duplication of preexisting $\beta$ cells (18). The mechanisms that regulate $\beta$ cell duplication are not known. The postnatal period in rodent life between birth and weaning is characterized by a massive increase in $\beta$ cell mass. We show that this increase is primarily due to the replication of terminally differentiated $\beta$ cells and have begun to address the role of cell cycle regulators in $\beta$ cell replication. Here we show that one specific D-cyclin, cyclin D2, plays an essential role in regulating the replication of terminally differentiated $\beta$ cells in the postnatal period. In cyclin $D 2^{-/-}$mice, the absence of endocrine cell replication results in a 3 -fold decrease in $\beta$ cell mass. Several lines of evidence indicate that the requirement of cyclin D2 is specific to endocrine cells. First, during this postnatal period, the body weight and size of the $c y c l i n D 2^{-/-}$mice do not dif-

A

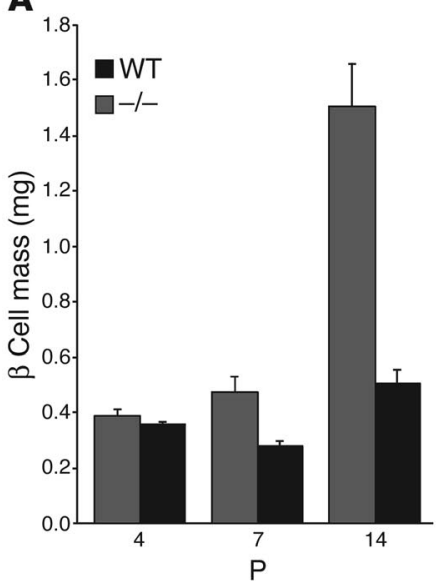

B
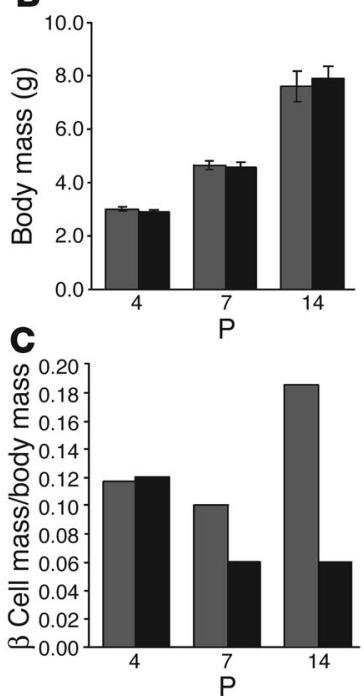
A

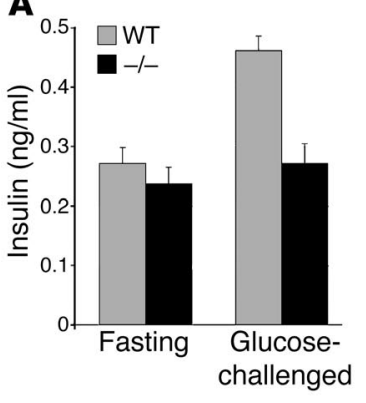

B

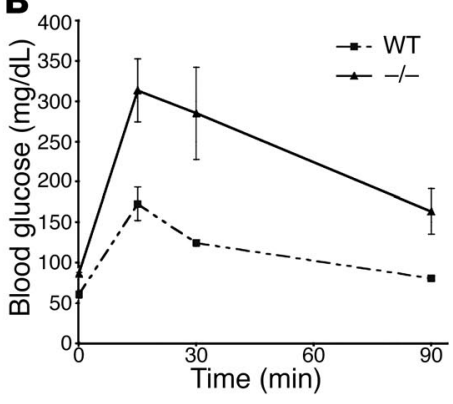

\section{Figure 6}

Cyclin D2 mutant mice have decreased plasma insulin levels and impaired glucose tolerance. (A) Plasma insulin levels in WT and cyclin $D 2^{-/-}$mice following overnight fasting and 30 minutes after glucose challenge ( $2 \mathrm{~g} / \mathrm{kg}$ body weight). Results are expressed as mean values \pm SEM of four WT and cyclin D2-l- mice. (B) Blood glucose levels were measured after 16-hour fasting (time 0 ) and after glucose injection ( $2 \mathrm{~g} / \mathrm{kg}$ body weight) in 12-week-old WT and cyclin $\mathrm{D} 2^{-1-}$ mice. Results are expressed as mean values \pm SEM of three WT and cyclin D2-/- mice.

fer from their WT littermates. Second, the weight of the pancreata from cyclin $\mathrm{D} 2^{-1-}$ mice did not differ from their WT littermates. Third, the replication of exocrine and ductal cells is unperturbed in the cyclin D2-/- mice. Thus, the absence of cyclin D2 leads to a narrow and highly selective consequence in the formation of the endocrine pancreas during postnatal development.

The selective replication defect in the endocrine pancreas is surprising because genetic analyses in mice indicate that in the overwhelming majority of cell types upregulation of the remaining D-cyclins can compensate for the loss of any particular D-cyclin (19). The failure to detect expression of other D-cyclins in $\beta$ cells in the cyclin $D 2^{-/-}$mice suggests that the lack of $\beta$ cell replication can be attributed to the inability of these $\beta$ cells to upregulate the remaining $\mathrm{D}$-cyclins. The failure to upregulate other D-cyclins in the $\beta$ cells of $c y c l i n D 2^{-/-}$mice is limited, however, to the early postnatal period since cyclin D1 expression is readily observed in the nuclei of islet cells 2 weeks after birth. The upregulation of cyclin D1 coincides with the transition of the endocrine pancreas from high rates of $\beta$ cell replication and expansion of $\beta$ cell mass in the 2 weeks after birth to steady-state levels of $\beta$ cell mass. Our results also indicate that the inability of $\beta$ cells to upregulate other $\mathrm{D}$-cyclins in early postnatal development indicates that the mitotic signaling that mediates high rates of $\beta$ cell replication impinges specifically on cyclin D2. This rigid wiring of the signaling pathway to the cell cycle machinery in endocrine islet cells may offer an opportunity for selective therapeutic intervention in diabetes by providing a means to regulate $\beta$ cell mass.

The D type cyclins act as sensors to mitotic stimuli and associate with partner cyclin-dependent kinases CDK4 and CDK6 to drive phosphorylation and subsequent inactivation of the retinoblastoma gene product, $\mathrm{pRb}$ and $\mathrm{pRb}$-related proteins $(16$, $17,20)$. The CDK4-null mice also display a defect in $\beta$ cell proliferation, indicating that CDK4 acts as the requisite partner for cyclin D2 in controlling cell cycle progression in $\beta$ cells (21). The CDK4null mice are $40 \%$ smaller than WT mice, however, signifying that CDK4 plays a more general role and is required in establishing homeostatic cell numbers throughout the animal (22). This indicates that the mitogenic signaling that regulates $\beta$ cell replication is likely to be mediated through cyclin D2. Thus, cyclin D2 may serve a crucial role in $\beta$ cells by acting as a sensor for mitogen signaling and linking to the cell cycle machinery.

An interest in identifying key components of the mitogenic pathway that regulates endocrine cell replication is to use them for in vitro propagation of $\beta$ cells or to devise ways to increase $\beta$ cell mass in vivo. Forcing endocrine cells to reenter the cell cycle can trigger programmed cell death, however. For example, overexpression of c-myc in $\beta$ cells leads to an initial increase in proliferation, fol- lowed by apoptosis and subsequently a depletion of the $\beta$ cell mass (23). This suggests that an intrinsic mechanism operates to protect the endocrine cells against improper activation of mitotic stimuli. Thus, it will be essential to understand how replication of endocrine cells without concomitant cell death is regulated during early postnatal development. A better understanding of how the cell cycle progression of $\beta$ cells is regulated could lead to new strategies for the therapy of diabetes.

\section{Methods}

Animal breeding and genotyping. Targeted disruption of the cyclin D2 allele has been described previously (24). Since homozygous cyclin D2-/- mice are infertile, the line was maintained in heterozygotes on a C57BL/6J background. Mice lacking cyclin D2 gene function were obtained from crosses of cyclin $D 2^{+/-}$animals and were compared with heterozygous and WT littermates. The day of birth was designated P0. DNA extracted from tails was used for PCR-based genotyping using standard methods. Primers used for genotyping were as follows: genomic cyclin D2 (5'-GCTGGCCTCCAATTCTAATC-3'); WT-specific (5'-CCAGATTTCAGCTGCTTCTG-3'); and KO-specific (5'CTAGTGAGACGTGCTACTTC- $\left.3^{\prime}\right)$. Embryos isolated from these mice were considered to be at 0.5 days of gestation at noon of the day the plugs were detected. Pancreatic tissue was dissected in cold PBS, fixed in $4 \%$ formaldehyde for 4 hours to overnight, followed by dehydration in grades of ethanol, and stored at $-20^{\circ} \mathrm{C}$ until processed for paraffin embedding.

RNA isolation and RT-PCR. The pancreata were dissected in cold PBS and homogenized in Tri Reagent (Molecular Research Center Inc.), and the total RNA was prepared according to the manufacturer's methods (25). RT-PCR was performed as described previously (26). The cDNA was synthesized from RNA using a standard reverse-transcription reaction. The cDNA was amplified using PCR MasterMix (Qiagen Inc.) and the following thermal cycler protocol: $94^{\circ} \mathrm{C}$ denaturation for 2 minutes, 25 cycles of $55^{\circ} \mathrm{C}$ annealing for 1 minute 30 seconds, $72^{\circ} \mathrm{C}$ extension for 1 minute, $93^{\circ} \mathrm{C}$ denaturation for 1 minute, and a final extension at $72^{\circ} \mathrm{C}$ for 5 minutes. Primers used were cyclin D1, F-CCGTATCTTACTTCAAGTGC; cyclin D1, R-ACTTGATGACTCTGGAAAGA; cyclin D2, F-CAGAACCTGTTGACCATC; cyclin D2, R-GCAAACTTGAAGTCGGTA; cyclin D3, F-GATTACACCTTTGCGATG; cyclin D3, R-TGGTGTGTGGATTTTACC.

Immunohistochemistry. The pancreata were oriented during the paraffinembedding process such that sections were cut along the head-tail axis. Sections were deparaffinized in toluene, rehydrated in grades of alcohol, and washed in $\mathrm{H}_{2} \mathrm{O}$. All slides were subject to antigen-retrieval protocols using antigen unmasking buffer (Vector Laboratories). After antigen unmasking, the slides were cooled to room temperature, permeabilized in $0.2 \%$ Triton X-100/TBS for 20 minutes, and blocked with $0.2 \%$ Tween 20/3\% IgG-free BSA/TBS. Primary Ab's were diluted in the blocking solution at the following dilutions: mouse anti-glucagon, 1:1,000 (SigmaAldrich); rabbit anti-cyclin D2, 1:4,000 (Santa Cruz Biotechnology Inc.); 
guinea pig anti-insulin 1:500 (Dako Corp.); rabbit anti-cleaved caspase-3, 1:200 (Cell Signaling Technology); cyclin D1, 1:800 (Santa Cruz Biotechnology Inc.); cyclin D3, 1:200 (Santa Cruz Biotechnology Inc.). Donkeyand goat-derived secondary Ab's conjugated to FITC, Cy3, or HRP were diluted 1:500 (Jackson ImmunoResearch Laboratories Inc.). HRP reactions were developed using DAB-Black Substrate Kit (Zymed Laboratories Inc.). All slides were mounted with Vectashield (Vector Laboratories) or Permount (Fisher Scientific Co.). Fluorescent slides were viewed using a Leica DXMRA microscope and images acquired using Openlab software.

BrdU incorporation. Thymidine analogue, BrdU (0.025 g per gram of body weight), was injected intraperitoneally 2 hours before harvesting the pancreata. Pancreata were isolated and processed for histology as described above. Mouse anti-BrdU Ab/nuclease solution (Amersham Biosciences) was applied for 1 hour.

$\beta$ cell mass analysis. The pancreata were trimmed of all nonpancreatic tissue, weighed, and processed for histology. The pancreata were embedded in paraffin such that longitudinal sections from tail to head of the pancreas were obtained. Ten representative sections from each pancreas (spanning the width of the pancreas) were used in the analysis of $\beta$ cell mass. Sections were deparaffinized in toluene and incubated in $0.3 \%$ hydrogen peroxide in ethanol for 30 minutes. Sections were washed in PBS and incubated in guinea pig anti-insulin 1:500 (Dako Corp.) overnight. A polyclonal guinea pig anti-porcine insulin primary $\mathrm{Ab}$ was used, followed by a peroxidase-labeled secondary Ab, developed with 3,3 diamino benzidine (DAB; Sigma-Aldrich) and counterstained with hematoxylin. An image of each slide was captured using Pathscan Enabler (Meyer Instruments Inc.) and Polaroid SprintScan $4000+$ with Polacolor Insight Software (Polaroid Corp.). Tissue areas were determined by marking the threshold of the captured image for brown staining ( $\beta$ cells) and total brown and blue stain (total tissue) using Metamorph software (Universal Imaging Corp.). The relative cross-sectional area of $\beta$ cells was determined by quantification of the cross-sectional area occupied by $\beta$ cells divided by the cross-sectional area of total tissue. Each section was analyzed to estimate $\beta$ cell and total tissue area. The $\beta$ cell mass per pancreas was estimated as the product of the relative cross-sectional area of $\beta$ cells per total tissue and the weight of the pancreas. The $\beta$ cell mass was calculated by examining pancreata from four animals for each age and genotype.

GTT and plasma insulin levels. Following a 14- to 16-hour fast, baseline blood glucose levels $(\mathrm{mg} / \mathrm{dl})$ were measured in tail vein blood from mice using OneTouch Ultra Glucose Meter (Lifescan Inc.). Glucose (2 mg dextrose/g body weight) in sterile PBS was injected intraperitoneally and blood glucose measured 15, 30, and 90 minutes after injection. GTTs were performed at 4,8 , and 12 weeks after birth. To measure plasma insulin levels, approximately $40 \mu \mathrm{l}$ blood was collected from the tail vein prior to and 30 minutes after intraperitoneal injection with glucose ( $2 \mathrm{mg} / \mathrm{g}$ body weight). Blood samples were centrifuged, and serum was used to measure insulin concentrations with an ELISA Rat/Mouse Insulin Kit (Linco Research Inc.).

\section{Acknowledgments}

We thank P. Butler and R. Scharfmann for comments and P. Sicinski for the cyclin D2 mice. We also acknowledge R. Soliz's assistance with histology and immunohistochemistry. This work was supported in part by start-up grants from the McAlister Endowment at Children's Hospital Los Angeles, the Larry L. Hillblom Foundation, and the NIH (R01 DK-68763, to A. Bhushan).

Received for publication May 7, 2004, and accepted in revised form August 10, 2004.

Address correspondence to: Anil Bhushan, Department of Biochemistry and Molecular Biology, University of Southern California, Developmental Biology Program, Saban Research Institute, Children's Hospital Los Angeles, 4560 Sunset Boulevard, Mailstop 35, Los Angeles, California 90027, USA. Phone: (323) 669-5558; Fax: (323) 671-3613; E-mail: abhushan@chla.usc.edu.

A. Bhushan's present address is: Hillblom Islet Research Center, University of California, Los Angeles, Los Angeles, California, USA.
1. Bruning, J.C., Winnay, J., Cheatham, B., and Kahn, C.R. 1997. Differential signaling by insulin receptor substrate 1 (IRS-1) and IRS-2 in IRS-1-deficient cells. Mol. Cell. Biol. 17:1513-1521.

2. Accili, D. 2001. A kinase in the life of the $\beta$ cell. J. Clin. Invest. 108:1575-1576. doi:10.1172/JCI200114454.

3. Bonner-Weir, S. 2000. Islet growth and development in the adult. J. Mol. Endocrinol. 24:297-302.

4. Lingohr, M.K., Buettner, R., and Rhodes, C.J. 2002. Pancreatic beta-cell growth and survival--a role in obesity-linked type 2 diabetes? [review]. Trends Mol. Med. 8:375-384.

5. Butler, A.E., et al. 2003. Beta-cell deficit and increased beta-cell apoptosis in humans with type 2 diabetes. Diabetes. 52:102-110.

6. Flier, S.N., Kulkarni, R.N., and Kahn, C.R. 2001 Evidence for a circulating islet cell growth factor in insulin-resistant states. Proc. Natl. Acad. Sci. U. S. A. 98:7475-7480.

7. Bonner-Weir, S. 2000. Perspective: Postnatal pancreatic beta cell growth [review]. Endocrinology. 141:1926-1929.

8. Edlund, H. 2002. Pancreatic organogenesis-developmental mechanisms and implications for therapy. Nat. Rev. Genet. 3:524-532.

9. Wilson, M.E., Scheel, D., and German, M.S. 2003. Gene expression cascades in pancreatic development. Mech. Dev. 120:65-80.

10. Gu, G., Dubauskaite, J., and Melton, D.A. 2002. Direct evidence for the pancreatic lineage: NGN3+ cells are islet progenitors and are distinct from duct progenitors. Development. 129:2447-2457.

11. Jensen, J., et al. 2000. Independent development of pancreatic alpha- and beta-cells from neurogenin 3 -expressing precursors: a role for the notch pathway in repression of premature differentiation. Diabetes. 49:163-176.

12. Bonner-Weir, S. 2000. Life and death of the pancreatic beta cells. Trends Endocrinol. Metab. 11:375-378.

13. Finegood, D.T., Scaglia, L., and Bonner-Weir, S. 1995. Dynamics of beta-cell mass in the growing rat pancreas. Estimation with a simple mathematical model. Diabetes. 44:249-256.

14. Svenstrup, K., Skau, M., Pakkenberg, B., Buschard, K., and Bock, T. 2002. Postnatal development of beta-cells in rats. Proposed explanatory model. APMIS. 110:372-378.

15. Sherr, C.J. 1995. Mammalian G1 cyclins and cell cycle progression. Proc. Assoc. Am. Physicians. 107:181-186.

16. Matsushime, H., et al. 1994. D-type cyclin-dependent kinase activity in mammalian cells. Mol. Cell. Biol. 14:2066-2076.

17. Bates, S., et al. 1994. CDK6 (PLSTIRE) and CDK4 (PSK-J3) are a distinct subset of the cyclin-dependent kinases that associate with cyclin D1. Oncogene. 9:71-79.

18. Dor, Y., Brown, J., Martinez, O.I., and Melton, D.A 2004. Adult pancreatic beta-cells are formed by self-duplication rather than stem-cell differentiation. Nature. 429:41-46.
19. Ciemerych, M.A., et al. 2002. Development of mice expressing a single D-type cyclin. Genes Dev. 16:3277-3289.

20. Matsushime, H., et al. 1992. Identification and properties of an atypical catalytic subunit (p34PSK$\mathrm{J} 3 / \mathrm{cdk} 4$ ) for mammalian D type G1 cyclins. Cell. 71:323-334.

21. Rane, S.G., et al. 1999. Loss of Cdk4 expression causes insulin-deficient diabetes and Cdk4 activation results in beta-islet cell hyperplasia. Nat. Genet. 22:44-52.

22. Martin, J., et al. 2003. Genetic rescue of Cdk4 null mice restores pancreatic beta-cell proliferation but not homeostatic cell number. Oncogene. 22:5261-5269.

23. Laybutt, D.R., et al. 2002. Overexpression of c-Myc in beta-cells of transgenic mice causes proliferation and apoptosis, downregulation of insulin gene expression, and diabetes. Diabetes. 51:1793-1804.

24. Sicinski, P., et al. 1996. Cyclin D2 is an FSH-responsive gene involved in gonadal cell proliferation and oncogenesis. Nature. 384:470-474.

25. Chomczynski, P., and Sacchi, N. 1987. Single-step method of RNA isolation by acid guanidinium thiocyanate-phenol-chloroform extraction. Anal. Biochem. 162:156-159.

26. Bhushan, A., Chen, Y., and Vale, W. 1998. Smad7 inhibits mesoderm formation and promotes neural cell fate in Xenopus embryos. Dev. Biol. 200:260-268. 